Olfactory ensheathing cells are the main phagocytic cells that remove axon debris during early development of the olfactory system

Lynnmaria Nazareth $^{1,2}$, Katie E. Lineburg ${ }^{3}$, Meng Inn Chuah ${ }^{4}$, Johana Tello Velasquez ${ }^{2}$, Fatemeh Chehrehasa ${ }^{1,2}$, James A. St John ${ }^{2 *}$, Jenny A.K. Ekberg ${ }^{1,2^{*}}$

${ }^{1}$ School of Biomedical Sciences, Queensland University of Technology, Brisbane, 4000, Queensland, Australia

${ }^{2}$ Eskitis Institute for Drug Discovery, Griffith University, Nathan, 4111, Queensland, Australia

${ }^{3}$ QIMR-Berghofer Medical Research Institute, Herston, 4006, Queensland, Australia

${ }^{4}$ School of Medicine, University of Tasmania, Hobart, 7005, Tasmania, Australia

Abbreviated title: Olfactory glia phagocytose axonal debris

Associate Editor: Thomas Finger

Key words: Olfactory nerve; neuron; apoptosis; bulbectomy; degeneration

*Corresponding authors:

Jenny Ekberg, School of Biomedical Sciences, Queensland University of Technology, Brisbane 4000, Queensland, Australia. Phone: +61 73138 0165, email: jenny.ekberg@qut.edu.au

James St John, Eskitis Institute for Drug Discovery, Griffith University, Nathan 4111, Queensland, Australia. Phone: +61 73735 3660, email: j.stjohn@ griffith.edu.au

\title{
Grant Information
}

This work was supported by funding from Griffith University to JStJ and JE and by an Australian Research Council Postdoctoral Fellowship, DP0986294, to JE. 


\section{Abstract}

During development of the primary olfactory system, axon targeting is inaccurate and axons inappropriately project within the target layer or over-project into the deeper layers of the olfactory bulb. As a consequence there is considerable apoptosis of primary olfactory neurons during embryonic and postnatal development and axons of the degraded neurons need to be removed. Olfactory ensheathing cells (OECs) are the glia of the primary olfactory nerve and are known to phagocytose axon debris in the adult and postnatal animal. However it is unclear when phagocytosis by OECs first commences. We have investigated the onset of phagocytosis by OECs in the developing mouse olfactory system by utilizing two transgenic reporter lines: OMP-ZsGreen mice which express bright green fluorescent protein in primary olfactory neurons and S100ß-DsRed mice which express red fluorescent protein in OECs. In crosses of these mice, the fate of the degraded axon debris is easily visualized. We found evidence of axon degradation at E13.5. Phagocytosis of the primary olfactory axon debris by OECs was first detected at E14.5. Phagocytosis of axon debris continued into the postnatal animal during the period when there was extensive mis-targeting of olfactory axons. Macrophages were often present in close proximity to OECs but they contributed only a minor role to clearing the axon debris, even after widespread degeneration of olfactory neurons by unilateral bulbectomy and methimazole treatment. These results demonstrate that from early in embryonic development OECs are the primary phagocytic cells of the primary olfactory nerve. 


\section{Introduction}

During development of the olfactory system, guidance of the axons that arise from the olfactory sensory neurons is not always accurate. For successful innervation, the sensory axons should terminate in the glomerular layer of the olfactory bulb, however many axons project to inappropriate regions of glomerular layer or over-extend into the deeper layers of the olfactory bulb (Graziadei et al., 1980; Santacana et al., 1992; Gong and Shipley, 1995; Tenne-Brown and Key, 1999; Chan et al., 2011). Without making successful connections with the target tissue these misguided axons are pruned or the neurons undergo apoptosis (Voyron et al., 1999; Cowan and Roskams, 2004) and the resultant cell debris needs to be removed. However, the cells responsible for clearing axonal debris during early development of the olfactory system have not been identified.

In adult mice it has been shown that olfactory ensheathing cells (OECs), rather than macrophages, are primarily responsible for clearing debris from primary sensory axons that arises during normal turnover of olfactory sensory neurons or after major injury ( $\mathrm{Su}$ et al., 2013). OECs are the specialized glia of the olfactory nerve and they ensheathe the axon fascicles from the olfactory mucosa up to their targets in the olfactory bulb and assist axon growth (Doucette, 1984; Chuah and Zheng, 1992; Ramon-Cueto and Valverde, 1995; Tennent and Chuah, 1996; Windus et al., 2011; Ekberg et al., 2012). During development, precursors of OECs arise from the neural crest and populate the olfactory placode from where they emerge to populate the olfactory nerve (Barraud et al., 2010) As the first olfactory sensory axons emerge from the basal layer of the olfactory epithelium, the OECs migrate ahead of, and ensheathe, the growing axons (Tennent and Chuah, 1996; Miller et al., 2010b; Miller et al., 2010a). Loss of OECs from the developing olfactory nerve results in fewer 
olfactory neurons and poor axon growth (Barraud et al., 2013) and thus OECs are crucial for the growth and guidance of primary olfactory axons during early development.

However, despite the suite of axon guidance molecules and the presence of OECs that assist the growth of axons during normal development, olfactory axon targeting is often inaccurate as has been clearly shown in previous studies (Graziadei et al., 1980; Santacana et al., 1992; Gong and Shipley, 1995; Tenne-Brown and Key, 1999; Chan et al., 2011). For example, in postnatal rats, more than one-third of axons project to multiple targets or over-project into the deeper layers (Tenne-Brown and Key, 1999) which indicates that there is a need for wide scale pruning and clearing of axonal debris during development. When axon guidance or normal function of neurons is perturbed, olfactory sensory axons also over-project into the deeper layers of the olfactory bulb particularly during early development (Montag-Sallaz et al., 2002; Walz et al., 2002; Cloutier et al., 2004; St John and Key, 2005) and ultimately need to be removed.

Which cells are responsible for removing the axonal debris from within the lamina propria and nerve fiber layer during development? OECs ensheathe the axon fascicles from the olfactory mucosa up to their targets in the olfactory bulb (Doucette, 1984; Chuah and Zheng, 1992) and are in constant contact with the growing axons placing them in the prime position for removing cell debris that arises from degradation of the misguided axons. Nevertheless, within this rapidly changing and developing environment, other cells such as macrophages may be responsible for clearing the cell debris. To determine which cells are responsible for clearing axonal debris within the developing olfactory system, we took advantage of a transgenic mouse reporter line which we previously generated, OMP-ZsGreen mice (Ekberg et al., 2011). In these mice, the bright and stable green fluorescent protein ZsGreen is 
expressed in primary olfactory sensory neurons and can be easily visualized after cellular degradation. We have previously used these transgenic mice to show that during early development, olfactory sensory axons that project past their target layer and enter the deeper layers of the olfactory bulb are phagocytosed by the radial glia that populate the central region of the olfactory bulb (Amaya et al., 2014). We have now examined the fate of degraded axons within the developing olfactory nerve fascicles and nerve fiber layer. 


\section{Materials and Methods}

\section{Animal strains}

Three different transgenic reporter mouse lines were used: (1) OMP-ZsGreen mice, in which the olfactory marker protein promoter drives expression of ZsGreen fluorescent protein in primary olfactory sensory neurons (Windus et al., 2007; Ekberg et al., 2011); (2) S100ßDsRed mice in which the human S100ß promoter drives expression of DsRed fluorescent protein in cells that express S100ß including OECs (Windus et al., 2007); and (3) Csf1rGal4VP16/UAS-ECFP mice (MacBlue mice) in which a modified promoter of the Csflr gene drives expression of Gal4VP16 transcriptional activator in macrophages and together with the Gal4-dependent promoter drives expression of ECFP in monocytes, tissue macrophages and microglia (Ovchinnikov et al., 2008). OMP-ZsGreen and S100ß-DsRed mice were previously generated in our laboratory; MacBlue mice were provided by D. Ovchinnichov. Crosses of OMP-ZsGreen and S100ß-DsRed were used for most experiments, and crosses of S100ßDsRed and MacBlue mice were used for experiments shown in Figure 4. All procedures were carried out with the approval of the Griffith University Animal Ethics Committee under the guidelines of the National Health and Medical Research Council of Australia and the Australian Commonwealth Office of the Gene Technology Regulator.

Surgical ablation of olfactory bulb (unilateral bulbectomy)

Unilateral bulbectomies were performed on postnatal day 4.5 using our previously established method (Chehrehasa et al., 2006). Pups were anaesthetized by placing them on ice for 8 min (Danneman and Mandrell, 1997) and then a midline incision was made to expose the cranial bones. The bone directly over the olfactory bulb was removed using a surgical drill with a $0.5 \mathrm{~mm}$ burr. The entire olfactory bulb was aspirated using a pulled glass pipette 
attached to a vacuum pump. The skin covering the site was sutured and the anti-inflammatory drug Flunix was administered via intramuscular injection (active ingredient flunixin, $1 \mathrm{mg} / \mathrm{kg}$ body weight; Parnell Laboratories, Australia). Animals were allowed to recover for three days. All procedures were carried out with the approval of, and in accordance with, the Griffith University Animal Ethics Experimentation Committee. Three pups were harvested three days after surgery at P7.

\section{Methimazole}

Widespread degeneration of olfactory neurons was induced using a method we have previously used (Chehrehasa et al., 2010) by giving postnatal day 4 pups a single intraperitoneal injection of methimazole $(50 \mathrm{mg} / \mathrm{kg}, 10 \mathrm{mg} / \mathrm{ml}$ in phosphate-buffered saline [PBS]). Three pups were harvested three days later at P7.

\section{Tissue preparation}

To harvest embryos, pregnant mice were euthanized by cervical dislocation, embryos were removed and decapitated. Postnatal pups were decapitated and adult mice were subjected to rising carbon dioxide asphyxiation. The tissues were fixed in $4 \%$ paraformaldehyde (PFA) in PBS, $\mathrm{pH} 7.4$, for $1 \mathrm{~h}$ at room temp or overnight at $4{ }^{\circ} \mathrm{C}$ and then cryoprotected in $30 \%$ sucrose in PBS with $0.1 \%$ azide at $4{ }^{\circ} \mathrm{C}$. Heads were placed in embedding matrix (O.C.T, Tissue-Tek) and snap frozen by immersion into 2-methyl butane which had being cooled with liquid nitrogen. Serial sagittal or coronal sections $(30 \mu \mathrm{m})$ were cut, mounted on slides and stored at $-20{ }^{\circ} \mathrm{C}$ before processing for immunohistochemistry. 
OECs were isolated from the lamina propria lining the nasal septum of E14.5 OMP-ZsGreen x S100ß-DsRed embryos. Cells were dissociated using Tryple Express (Life Technologies) into single cell suspension, plated into 8-well glass coverslip bottomed plates (Sarstedt) in medium containing Dulbecco's modified Eagle medium (DMEM), 10\% fetal bovine serum (Bovogen), G5 supplement (Life Technologies), gentamicin, Glutamax (Life Technologies) and incubated in $5 \% \mathrm{CO}_{2}$ at $37^{\circ} \mathrm{C}$ for $6 \mathrm{~h}$ to allow attachment of cells to the plate surface. Live cells were then imaged using confocal microscopy after which cells were then grown overnight and fixed with 4\% PFA before immunostaining as described below. Tissue was dissected from 6 animals.

In vitro phagocytosis of axon debris by OECs

Dissociated cultures of OECs were prepared from S100ß-DsRed E14.5 pups and plated onto 8-well glass coverslip bottom culture dishes. Axonal debris was generated by dissecting out the nerve fiber layer of the olfactory bulb of an OMP-ZsGreen mouse and partially digesting it in TrypLE Express (Life Technologies) and collagenase $(0.1 \mathrm{mg} / \mathrm{ml}$, Life Technologies) for 30 min. The reaction was stopped by addition of fetal bovine serum (Bovogen), centrifuged for 5 min and resuspended in DMEM and triturated using a syringe with a 27 gauge needle. The axonal debris (identifiable by expression of ZsGreen protein) was added to dissociated cultures of DsRed OECs. Prior to addition of the ZsGreen axon debris, live cells were initially imaged using epifluorescent CellR microscope (Olympus). After addition of the ZsGreen axon debris, live cells were imaged using laser scanning confocal microscopy as described below.

\section{Immunochemistry}


Immunohistochemistry was performed on tissue sections and cell cultures using our previously established methods (Windus et al., 2007; Windus et al., 2011; Chehrehasa et al., 2012). The samples were blocked with $2 \%$ bovine serum albumin (BSA) in $0.1 \mathrm{M}$ PBS with $0.03 \%$ Triton-X (BSA/PBS/Tx) for $30 \mathrm{~min}$ at room temp, then incubated with the primary antibodies as specified in Table 1 diluted in BSA/PBS/Tx overnight at $4{ }^{\circ} \mathrm{C}$. Samples were then washed three times with PBS/TX and incubated with the secondary antibodies diluted in BSA/PBS/Tx for $1 \mathrm{~h}$ at room temp. Cell nuclei were stained with 4'6-diamidino-2pheylindole (DAPI). The antibodies used were: polyclonal Iba1 (Garcia-Ovejero et al., 2013), p75ntr (Tripathi and McTigue, 2008), Lamp1 (Stewart et al., 2012), Gap43 (Rivard et al., 2009); followed by the secondary antibodies anti-rabbit Alexa Fluor 647 (5 $\mu \mathrm{g} / \mathrm{mL}$; Life Technologies, A-21244, RRID:AB_10561706).

\section{Quantification of macrophages}

Coronal cryostat sections $(30 \mu \mathrm{m}$ thick) of postnatal day 7 pups from crosses of S100ßDsRed and MacBlue mice were imaged using confocal microscopy to obtain z-stacks of optical slices covering a depth of $15 \mu \mathrm{m}$. For each of three animals, three sections that were separated by $240 \mu \mathrm{m}$ from the previous section (covering a total of $480 \mu \mathrm{m}$ in the rostralcaudal plane) were examined. Macrophages were identified by expression of cyan fluorescent protein (CFP). The distribution of CFP enabled easy visualisation of the cell body which expressed high levels of CFP; the multiple processes which extended from the cell body and projected into the surrounding tissue had less intense expression of CFP. Thus the cell body was defined as the central region of intense CFP expression from which processes extended that had less intense CFP expression. Only cells in which the cell body was present and entirely within the region of interest measuring $200 \mu \mathrm{m}$ square $\left(40,000 \mu \mathrm{m}^{2}\right)$ were counted. Statistical analysis performed using Student's t-test. 
Scanning electron microscopic analysis of OEC reaction to axon debris

Dissociated cultures of postnatal OECs had the medium replaced with either control medium or medium containing axon debris as prepared and described above and were then incubated for up to $2 \mathrm{~h}$ and then fixed in $4 \%$ PFA for $10 \mathrm{~min}$. Specimens were washed twice in PBS, post-fixed in $1 \%$ osmium tetroxide $(10 \mathrm{~min})$, rinsed in PBS and left in $70 \%$ ethanol until ready for dehydration. Specimens were dehydrated in an increasing series of methanols (70$100 \%$ ), then transferred to dry acetone. They were then critical point dried, sputter coated with gold (Balzers) and examined on a scanning electron microscope (JEOL JSM-840). The number of pseudopodia protrusions per cell was counted and statistical analysis was performed using a two-way analysis of variance.

\section{Image capture}

Laser scanning confocal microscope images were taken using an Olympus FV1000 microscope and processed on FV10-ASW 3.01.01.09 Viewer software. In order to enhance presentation, brightness and contrast were adjusted uniformly across the field of view using the FV10-ASW 3.1 Viewer software and ImageJ 1.47t software. For images comparing treatments, the images were captured using the same confocal settings and were color balanced using the same brightness and contrast adjustments. Live cell epifluorescent imaging was performed using an Olympus CellR epifluorescent microscope. Panels were created by using Adobe Illustrator CS5 15.0.2. 


\section{Results}

To investigate the onset of phagocytosis by OECs we utilized OMP-ZsGreen transgenic mice in which the bright fluorescent protein ZsGreen is expressed in primary olfactory sensory neurons and their axons (Ekberg et al., 2011). In these mice, ZsGreen is expressed by olfactory sensory neurons from early in development of the olfactory system with numerous neurons expressing ZsGreen at embryonic day 11.5 (E11.5) (Fig. 1A). Immunolabelling with antibodies against Gap43 which is a marker of immature neurons showed that the majority of neurons that expressed Gap43 also expressed ZsGreen (Fig. 1B-D) although some neurons expressed Gap43 but did not express ZsGreen (arrowhead, Fig. 1B-D). At E13.5, ZsGreen was strongly expressed by the vast majority of neurons that were immunolabelled by Gap43 with only a few neurons expressing Gap43 but not ZsGreen (arrowhead, Fig. 2E-F). The ZsGreen provided distinct visualization of the axons of the olfactory neurons at this age (arrow with tail, Fig. 1E-F). We crossed the OMP-ZsGreen mice with S100ß-DsRed mice (Windus et al., 2007) which express the red fluorescent protein DsRed in OECs. In these mice the DsRed OECs are easily visualized as they surround the ZsGreen axons (Fig. 1G-H). Immunolabelling using antibodies against the OEC marker p75ntr showed colocalization with the DsRed OECs (Fig. 1G-I).

\section{Embryonic OECs phagocytose primary olfactory axon debris}

In E13.5 pups, the ZsGreen protein was strongly expressed by primary olfactory sensory neurons throughout the olfactory epithelium (Fig. 2A) as well as migrating neurons within the migratory mass (arrowhead, Fig. 2B) which are known to express OMP protein (Valverde et al., 1993; Tarozzo et al., 1994). The ZsGreen protein provided distinct visualization of individual axons such that axons that over-projected into the deeper layers of the olfactory bulb were easily observed (arrow, Fig. 2B). Small aggregates of ZsGreen fragments were 
present near the nerve fascicles (arrow with tail, Fig. 2B) but these did not co-localize with OECs (data not shown).

In E14.5 embryos from crosses of OMP-ZsGreen x S100ß-DsRed mice, primary olfactory axons that projected to the olfactory bulb made a more extensive nerve fiber layer (Fig. 2C) and numerous axons over-projected (arrow in Fig. 2D) past the nerve fiber layer into the deeper layers of the olfactory bulb. Close examination of the distribution of the ZsGreen protein within the external plexiform layer indicated that some of the axons that had overprojected were not intact or continuous, but instead appeared to be fragmented (arrowhead, Fig. 2D), suggesting that some mis-targeted axons were being degraded. At this age, the DsRed-expressing OECs were in close contact with the primary olfactory axons in the lamina propria (Fig. 2E) and the nerve fiber layer. We examined the OECs and axons within the lamina propria of E14.5 embryos to determine if axon debris was present and associated with OECs. Intact olfactory axons were easily visualized and had a uniform and continuous expression of ZsGreen (Fig. 2E). However, there were also numerous bright accumulations of ZsGreen-expressing axon debris within the fascicles (Fig. 2E). Some of the ZsGreen axon debris was localized within OECs (arrows, Fig. 2E) while some debris was localized external to OECs (arrowhead, Fig. 2E). A three-dimensional reconstruction of a laser scanning confocal microscope z-stack confirmed the ZsGreen axon debris was not continuous with intact axons and that some debris was contained within the OEC (Supplemental movie 1).

At E15.5, the nerve fiber layer was more extensive (Fig. 2F) and numerous axons overprojected into the deeper layer of the developing olfactory bulb (arrow, Fig. 2G). OECs surrounded the axons in the lamina propria and within the nerve fiber layer (Fig. 2G). Aggregations of ZsGreen axon debris were present in the axon fascicles within the lamina 
propria (Fig. 2H) and within the nerve fiber layer (Fig. 2I) with many of the aggregations of ZsGreen axon debris localized within OECs (arrows, Fig. 2H-I). Three-dimensional reconstruction of panel 2I confirmed the ZsGreen debris had accumulated within OECs (Supplemental movie 2). With increasing embryonic age, the mis-targeting of axons was more extensive (arrow, Fig. 2J). Within axon fascicles of the lamina propria and nerve fiber layer of E17.5 embryos, numerous OECs clearly contained accumulations of ZsGreen axon debris (arrows, Fig. 2K-L).

\section{Postnatal OECs phagocytose primary olfactory axon debris}

At postnatal day 3 (P3) pups, while many axons projected to their target glomeruli, numerous axons also continued to over-project into the deeper layers of the olfactory bulb (Fig. 3A-B) and fragmented axon debris was evident in the external plexiform layer (arrowhead, Fig. 3B). At this age, OECs in axon fascicles within the lamina propria (arrows, Fig. 3C-E) and in the nerve fiber layer (Fig. 3F, Supplemental movie 3) had internalized green fluorescent axon debris.

\section{Macrophages are not the principal phagocytic cells of olfactory axon debris}

We examined the distribution of macrophages by immunohistochemical staining with antibodies against Iba1. In postnatal day 7 pups, macrophages were generally located around the exterior of axon fascicles and the outer limit of the nerve fiber layer (arrows, Fig. 4A). Some macrophages were in close proximity to the axon fascicles and contained small amounts of axon debris (arrowhead, Fig. 4B). In contrast, OECs frequently contained large amounts of axon debris (arrow, Fig. 4B). Similarly, in the nerve fiber layer macrophages were infrequently present and sometimes contained axon debris (arrowhead, Fig. 4C) while OECs contained large amounts of debris (arrows, Fig. 4C). To further confirm the 
distribution of macrophages in relation to OECs, we crossed the S100ß-DsRed mice with MacBlue mice in which the macrophages express blue fluorescent protein (Ovchinnikov et al., 2008). Macrophages were present around the exterior of OECs and axon fascicles (Fig. 4D). Macrophages that were within the confines of a large fascicle were restricted to the exterior of the smaller bundles (arrowhead, Fig. 4D) within the large fascicle. In the nerve fiber layer, MacBlue macrophages were infrequently present and were usually located between bundles of axons (arrowhead, Fig. 4E). At lower magnification of the olfactory bulbs, it was apparent that macrophages were infrequently present in the nerve fiber layer whereas they were abundant within the deeper layers of the olfactory bulb (Fig. 4F-I). We quantified the number of macrophages per unit area in the nerve fiber layer and the adjacent external plexiform layer. Macrophages were four times more numerous in the external plexiform layer of the bulb compared to the nerve fiber layer ( $\mathrm{p}<0.001 ;$ Fig. $4 \mathrm{~J})$.

\section{Embryonic OECs phagocytose axon debris in vivo}

In order to determine whether the green fluorescent axon debris was internalized within embryonic OECs in vivo, we dissected out OECs from E14.5 OMP-ZsGreen x S100ß-DsRed embryos and plated them into culture dishes. Upon attachment to the culture dish we examined the dissociated OECs and found they contained the green fluorescent axon debris (Fig. 5A, arrows Fig. 5B) demonstrates that the OECs had internalized the green debris whilst in vivo. Immunostaining with antibodies against p75ntr confirmed the identity of the in vitro preparations of E14.5 OECs that had internalized ZsGreen axon debris (Fig. 5B-C). Phagocytosed cell debris was processed into lysosomes and immunostaining with lysosomalassociated protein 1 (lamp1) antibodies confirmed that axon debris colocalized with lysosomes (Fig. 5D-F). We next examined OECs dissected from E15.5 OMP-ZsGreen x S100ß-DsRed embryos immediately upon attachment to the culture plate. The E15.5 DsRed 
OECs contained numerous accumulations of ZsGreen debris within the cell body (arrows, Fig. 5G) and along the shaft of the process (arrowheads, Fig. 5H).

\section{OECs phagocytose axon debris in vitro}

To determine the response of OECs to axon debris in vitro, we cultured OECs from the lamina propria of E14.5 S100ß-DsRed embryos (not crossed with OMP-ZsGreen mice). Eighteen hours after plating, the OECs had a largely bipolar morphology with numerous dynamic lamellipodia protruding into the surrounding medium (arrowheads, Fig. 6A). ZsGreen fluorescent axon debris that had been prepared from the nerve fiber layer of OMPZsGreen mice was added to the culture medium. Laser scanning confocal microscopy of the live cells was performed $4.15 \mathrm{~h}$ after addition of the debris. The ZsGreen debris was internalized and present within the cell body of the OECs (arrows, Fig. 6B) and along the shaft of the process (arrowhead, Fig. 6B).

We next examined the reaction of the OECs to axon debris by using scanning electron microscopy to visualize the pseudopodial protrusions on the OECs. OECs in control medium had few pseudopodial protrusions (Fig. 6C) while OECs exposed to axon debris for 15 min had numerous pseudopodial protrusions (Fig. 6D). Quantification of the number of pseudopodial protrusions per cell body showed that after 5-15 min OECs had significantly more $(\mathrm{p}<0.001)$ protrusions compared to control OECs (Fig. 6E).

\section{OECs phagocytose primary olfactory axon debris after widespread damage}

Unilateral bulbectomy (surgical removal of one olfactory bulb) results in the death of neurons in the olfactory epithelium on the same side, while the neurons and olfactory bulb on the contralateral side are largely unaffected (Fig. 7A). Following the death of the olfactory 
neurons, the axons degenerate and must be removed by phagocytic cells. We performed the unilateral bulbectomy at $\mathrm{P} 4$ and allowed the animals to recover for three days to determine if OECs were responsible for the clearing of axon debris after large scale injury in the neonatal animal. On the contralateral (unablated/control) side, coronal sections revealed the normal morphology of OECs that ensheathed the axon fascicles within the lamina propria. The OECs extended numerous lamellipodia throughout the fascicle (Fig. 7B-E). The thin axons were in close contact with the OECs (Fig. 7C-D) and some debris was internalized within the OECs (arrows, Fig. 7C-D). Three-dimensional reconstruction of the control fascicle further revealed the extent of lamellipodia invasion into the fascicle and the close relationship with the axons (Fig. 7C, E, Supplemental movies 4 and 5). In contrast, on the bulbectomized side the OECs displayed distinctly different morphology compared to the contralateral control side (Fig. 7FJ). The neurons in the olfactory epithelium had degenerated as had the axons within the fascicles and there were large accumulations of axon debris within OECs (Fig. 7H-J). The OECs continued to surround the fascicles (arrowhead, Fig. 7G) however the lamellipodia were more restricted and were absent from much of the internal regions of the fascicle (arrows, Fig. 7G-H). Three-dimensional reconstruction revealed the open structure of the axon fascicles in the bulbectomized side (Fig. 7H, J, Supplemental movies 6 and 7). We determined if macrophages accumulated axon debris in the fascicles in bulbectomized side (Fig. 8A) using immunolabelling with anti-Iba1 antibodies (Fig. 8B). As in normal healthy fascicles (Fig. 4) macrophages were present around the exterior of the fascicles and some contained small amounts of axon debris (arrowheads, Fig. 8B), however the OECs contained the majority of axon debris (arrows, Fig. 8B). We then used the methimazole model of olfactory neuron degeneration in which methimazole destroys the olfactory neurons within the epithelium (Fig. 8C). In methimazole treated P7 pups, macrophages were present around 
axon fascicles but contained little or no axon debris (arrowheads, Fig. 8D) while OECs contained large amounts of axon debris (arrows, Fig. 8D). 


\section{Discussion}

We have shown during early development of the primary olfactory system in mice that the OECs phagocytose axon debris that arises from the primary olfactory axons. Phagocytosis of axon debris by OECs was detected at E14.5 onwards which coincides with the period when primary olfactory axons are searching for their targets and often inappropriately over-project into the deeper layers of the olfactory bulb. While macrophages were present around the olfactory nerve fascicles, they were usually not in proximity to the axons and those that were did not accumulate large amounts of axon debris. After induction of widespread degeneration of primary olfactory neurons in the postnatal animal, OECs were the principal cells that phagocytosed the debris that arose from the degraded axons.

\section{OECs are the main phagocytic cell during early development}

It has been previously shown that OECs from the neonatal and adult rat are phagocytic cells which remove cell debris in vivo in the healthy olfactory system (Li et al., 2005; Wewetzer et al., 2005; Su et al., 2013) and after degeneration of the adult olfactory system (Su et al., 2013). In vitro assays have also demonstrated that OECs are phagocytic cells. In these assays postnatal rat OECs phagocytosed bacteria (Leung et al., 2008), adult mouse OECs phagocytosed olfactory neuron debris (Su et al., 2013), and we have previously shown that postnatal-derived mouse OECs can phagocytose bacteria in vitro (Panni et al., 2013). Our current results now show that OECs remove cell debris from as early as embryonic day 14.5. During development, primary olfactory axon targeting is not accurate with many axons projecting to inappropriate glomeruli or over-projecting past the glomerular layer and entering the deeper layers of the olfactory bulb. We have previously shown that primary olfactory axons that over-project into the external plexiform layer during early embryonic development are phagocytosed by radial glia (Amaya et al., 2014). However, many axons 
project to incorrect targets within the glomerular layer (Tenne-Brown and Key, 1999) and without successful innervation they are likely to be pruned or degraded. Apoptosis of olfactory neurons during development is quite widespread and is first detected in the mouse embryo from E12-E13 and continues into the postnatal animal (Voyron et al., 1999; Cowan and Roskams, 2004) consistent with the timing of inappropriate axon targeting and overprojection. In the adult, apoptosis of both immature and mature primary olfactory neurons occurs in the healthy animal further demonstrating that immature olfactory neurons undergo apoptosis (Holcomb et al., 1995). With such extensive and continuous apoptosis of the primary olfactory neurons, the debris arising from the degraded axons must be removed. The earliest we detected phagocytosis of axon debris by OECs was at E14.5. We did not detect axon debris within OECs at E13.5 despite the infrequent appearance of isolated ZsGreen fragments that appeared similar to those that were present at later ages and were internalized by OECs. Thus we conclude that the degradation of primary olfactory axons commences around E13.5 but that phagocytosis of the debris does not occur until E14.5.

\section{Minimal contribution by macrophages}

Macrophages are scavenging phagocytic cells in many regions of the body, but in the primary olfactory system they play a minor role in removing axon debris. Consistent with the distribution of macrophages in the adult olfactory system (Su et al., 2013), macrophages were present around the olfactory nerve fascicles that projected through the lamina propria, but they were infrequently found within the nerve fascicles. When they were within the nerve fascicles, it was usually within a large fascicle which comprised several smaller bundles that had coalesced and the macrophages were restricted to the regions between the smaller bundles. In the nerve fiber layer of the olfactory bulb, macrophages were infrequently found and were at a much lower density than in the adjacent external plexiform layer. It was clear 
that some macrophages had phagocytosed small amounts of axon debris in the lamina propria and nerve fiber layer, however numerous adjacent OECs had accumulated large amounts of debris and thus we conclude that macrophages made only a minor contribution to the removal of axon debris from the primary olfactory nerve pathway during development. Even after widespread degeneration of primary olfactory axons caused by unilateral bulbectomy or administration of methimazole, the macrophages in the postnatal mouse continued to play a minor role and OECs were responsible for phagocytosis of the vast amount of cell debris, which is consistent with the response of OECs in the injured adult olfactory system (Su et al., 2013).

The removal of axon debris by OECs, rather than relying on the infiltration of macrophages, likely provides a more rapid mechanism for maintaining an environment suitable for regenerating axons and adds another role by which OECs aid the regenerative ability of the olfactory system. The OECs maintain the structural integrity of the nerve fascicles by providing open continuous channels through which the regenerating axons are guided ( $\mathrm{Li}$ et al., 2005). The OECs also respond after injury by rapidly proliferating throughout the olfactory nerve pathway as well as proliferating from likely progenitors that line the basal layer of the olfactory epithelium and then migrating along the nerve fascicles (Chehrehasa et al., 2012). Thus OECs play multiple roles, from guiding the axons and maintaining the structure of the fascicles to removing axon debris.

\section{Morphology changes after widespread degeneration}

In the current study we showed that after unilateral bulbectomy, the axons rapidly degraded and that there was less extensive distribution of lamellipodia of the OECs throughout the fascicle. A previous study that examined the structure of OECs using electron microscopy 
suggested that OECs undergo little morphological change in response in denervation (Williams et al., 2004). However using the three-dimensional reconstruction of laser scanning confocal microscopy we found distinct changes at the level of the processes within the nerve fascicles which left more open channels within the fascicles. This is a potential route that can be taken advantage of by invading bacteria (Herbert et al., 2012). We previously showed that intranasal infection by the bacteria Burkholderia pseudomallei resulted in the death of the olfactory neurons. Subsequently, the degraded axons were removed from the fascicles leaving an open conduit through which the bacteria migrated up to the olfactory bulbs (St John et al., 2014). As macrophages are largely absent from the internal regions of the olfactory nerves even after widespread degeneration, the invading bacteria could avoid detection by the macrophages and migrate into the brain. While OECs could phagocytose the bacteria, the retraction of lamellipodia leaving open conduits within the fascicle would also aid the bacteria in evading the phagocytic OECs and facilitate their migration to the brain.

In summary, OECs are active phagocytic cells that remove axon debris from the primary olfactory nerves from as early as E14.5. Macrophages play a minor role in the removal of olfactory axon debris even after widespread degeneration of olfactory neurons. The OECs react to widespread degeneration of neurons by phagocytosing the axon debris and remodelling of their lamellipodia such that open channels are created within the axon fascicles. The rapid clearing of cell debris and open spaces through the fascicles likely promote new axon growth and may be a key factor in the regenerative ability of the olfactory system. 


\section{Conflict of Interest Statement}

The authors declare no conflict of interest.

\section{Role of Authors}

All authors had full access to all the data in the study and take responsibility for the integrity of the data and the accuracy of the data analysis. Study concept and design: JE, JStJ. Acquisition of data: LN, KL, JT, MC, JStJ. Statistical analysis: MC, JStJ. All authors contributed to the analysis and interpretation of data. Drafting of the manuscript: JStJ. Critical revision of the manuscript for important intellectual content: JStJ, JE, FC. Obtained funding: JE. JStJ. 


\section{Literature Cited}

Amaya DA, Wegner M, Stolt CC, Chehrehasa F, Ekberg JA, St John JA (2014) Radial glia phagocytose axonal debris from degenerating over-extending axons in the developing olfactory bulb. J Comp Neurol.

Barraud P, St John JA, Stolt CC, Wegner M, Baker CV (2013) Olfactory ensheathing glia are required for embryonic olfactory axon targeting and the migration of gonadotropinreleasing hormone neurons. Biol Open 2:750-759.

Barraud P, Seferiadis AA, Tyson LD, Zwart MF, Szabo-Rogers HL, Ruhrberg C, Liu KJ, Baker CV (2010) Neural crest origin of olfactory ensheathing glia. Proc Natl Acad Sci U S A 107:21040-21045.

Chan C, Ekberg JA, Lineburg KE, Scott SE, Chehrehasa F, Windus LC, Claxton C, MackaySim A, Key B, St John JA (2011) Correction of aberrant axon growth in the developing mouse olfactory bulb. Mol Cell Neurosci 46:282-295.

Chehrehasa F, St John JA, Key B (2006) Implantation of a scaffold following bulbectomy induces laminar organization of regenerating olfactory axons. Brain Res 1119:58-64.

Chehrehasa F, Ekberg JA, Lineburg K, Amaya D, Mackay-Sim A, St John JA (2012) Two phases of replacement replenish the olfactory ensheathing cell population after injury in postnatal mice. Glia 60:322-332.

Chehrehasa F, Windus LC, Ekberg JA, Scott SE, Amaya D, Mackay-Sim A, St John JA (2010) Olfactory glia enhance neonatal axon regeneration. Mol Cell Neurosci 45:277288.

Chuah MI, Zheng DR (1992) The human primary olfactory pathway: fine structural and cytochemical aspects during development and in adults. Microsc Res Tech 23:76-85.

Cloutier JF, Sahay A, Chang EC, Tessier-Lavigne M, Dulac C, Kolodkin AL, Ginty DD (2004) Differential requirements for semaphorin 3F and Slit-1 in axonal targeting, fasciculation, and segregation of olfactory sensory neuron projections. J Neurosci 24:9087-9096.

Cowan CM, Roskams AJ (2004) Caspase-3 and caspase-9 mediate developmental apoptosis in the mouse olfactory system. J Comp Neurol 474:136-148.

Danneman PJ, Mandrell TD (1997) Evaluation of five agents/methods for anesthesia of neonatal rats. Lab Anim Sci 47:386-395.

Doucette JR (1984) The glial cells in the nerve fiber layer of the rat olfactory bulb. Anat Rec 210:385-391.

Ekberg JA, Amaya D, Chehrehasa F, Lineburg K, Claxton C, Windus LC, Key B, MackaySim A, St John JA (2011) OMP-ZsGreen fluorescent protein transgenic mice for visualisation of olfactory sensory neurons in vivo and in vitro. J Neurosci Methods 196:88-98.

Ekberg JAK, Amaya D, Mackay-Sim A, St John JA (2012) The Migration of Olfactory Ensheathing Cells during Development and Regeneration. Neurosignals 20:147-158.

Garcia-Ovejero D, Arevalo-Martin A, Paniagua-Torija B, Sierra-Palomares Y, MolinaHolgado E (2013) A cell population that strongly expresses the CB1 cannabinoid receptor in the ependyma of the rat spinal cord. J Comp Neurol 521:233-251.

Gong Q, Shipley MT (1995) Evidence that pioneer olfactory axons regulate telencephalon cell cycle kinetics to induce the formation of the olfactory bulb. Neuron 14:91-101.

Graziadei GA, Stanley RS, Graziadei PP (1980) The olfactory marker protein in the olfactory system of the mouse during development. Neuroscience 5:1239-1252.

Herbert RP, Harris J, Chong KP, Chapman J, West AK, Chuah MI (2012) Cytokines and olfactory bulb microglia in response to bacterial challenge in the compromised primary olfactory pathway. J Neuroinflammation 9:109. 
Holcomb JD, Mumm JS, Calof AL (1995) Apoptosis in the neuronal lineage of the mouse olfactory epithelium: regulation in vivo and in vitro. Dev Biol 172:307-323.

Leung JY, Chapman JA, Harris JA, Hale D, Chung RS, West AK, Chuah MI (2008) Olfactory ensheathing cells are attracted to, and can endocytose, bacteria. Cell Mol Life Sci 65:2732-2739.

Li Y, Field PM, Raisman G (2005) Olfactory ensheathing cells and olfactory nerve fibroblasts maintain continuous open channels for regrowth of olfactory nerve fibres. Glia 52:245-251.

Miller AM, Treloar HB, Greer CA (2010a) Composition of the migratory mass during development of the olfactory nerve. J Comp Neurol 518:4825-4841.

Miller AM, Maurer LR, Zou DJ, Firestein S, Greer CA (2010b) Axon fasciculation in the developing olfactory nerve. Neural Dev 5:20.

Montag-Sallaz M, Schachner M, Montag D (2002) Misguided axonal projections, neural cell adhesion molecule 180 mRNA upregulation, and altered behavior in mice deficient for the close homolog of L1. Mol Cell Biol 22:7967-7981.

Ovchinnikov DA, van Zuylen WJ, DeBats CE, Alexander KA, Kellie S, Hume DA (2008) Expression of Gal4-dependent transgenes in cells of the mononuclear phagocyte system labeled with enhanced cyan fluorescent protein using Csf1r-Gal4VP16/UASECFP double-transgenic mice. J Leukoc Biol 83:430-433.

Panni P, Ferguson IA, Beacham I, Mackay-Sim A, Ekberg JA, St John JA (2013) Phagocytosis of bacteria by olfactory ensheathing cells and Schwann cells. Neuroscience letters 539:65-70.

Ramon-Cueto A, Valverde F (1995) Olfactory bulb ensheathing glia: a unique cell type with axonal growth-promoting properties. Glia 14:163-173.

Rivard RL, Birger M, Gaston KJ, Howe AK (2009) AKAP-independent localization of typeII protein kinase A to dynamic actin microspikes. Cell Motil Cytoskeleton 66:693709.

Santacana M, Heredia M, Valverde F (1992) Transient pattern of exuberant projections of olfactory axons during development in the rat. Brain Res Dev Brain Res 70:213-222.

St John JA, Key B (2005) Olfactory marker protein modulates primary olfactory axon overshooting in the olfactory bulb. J Comp Neurol 488:61-69.

St John JA, Ekberg JA, Dando SJ, Meedeniya AC, Horton RE, Batzloff M, Owen SJ, Holt S, Peak IR, Ulett GC, Mackay-Sim A, Beacham IR (2014) Burkholderia pseudomallei penetrates the brain via destruction of the olfactory and trigeminal nerves: implications for the pathogenesis of neurological melioidosis. mBIO 5:e00025.

Stewart RS, Teng H, Wilkinson RS (2012) "Late" macroendosomes and acidic endosomes in vertebrate motor nerve terminals. J Comp Neurol 520:4275-4293.

Su Z, Chen J, Qiu Y, Yuan Y, Zhu F, Zhu Y, Liu X, Pu Y, He C (2013) Olfactory ensheathing cells: the primary innate immunocytes in the olfactory pathway to engulf apoptotic olfactory nerve debris. Glia 61:490-503.

Tarozzo G, Peretto P, Perroteau I, Andreone C, Varga Z, Nicholls J, Fasolo A (1994) GnRH neurons and other cell populations migrating from the olfactory neuroepithelium. Ann Endocrinol (Paris) 55:249-254.

Tenne-Brown J, Key B (1999) Errors in lamina growth of primary olfactory axons in the rat and mouse olfactory bulb. J Comp Neurol 410:20-30.

Tennent R, Chuah MI (1996) Ultrastructural study of ensheathing cells in early development of olfactory axons. Brain Res Dev Brain Res 95:135-139.

Tripathi RB, McTigue DM (2008) Chronically increased ciliary neurotrophic factor and fibroblast growth factor-2 expression after spinal contusion in rats. J Comp Neurol 510:129-144. 
Valverde F, Heredia M, Santacana M (1993) Characterization of neuronal cell varieties migrating from the olfactory epithelium during prenatal development in the rat. Immunocytochemical study using antibodies against olfactory marker protein (OMP) and luteinizing hormone-releasing hormone (LH-RH). Brain Res Dev Brain Res 71:209-220.

Voyron S, Giacobini P, Tarozzo G, Cappello P, Perroteau I, Fasolo A (1999) Apoptosis in the development of the mouse olfactory epithelium. Brain Res Dev Brain Res 115:49-55.

Walz A, Rodriguez I, Mombaerts P (2002) Aberrant sensory innervation of the olfactory bulb in neuropilin-2 mutant mice. J Neurosci 22:4025-4035.

Wewetzer K, Kern N, Ebel C, Radtke C, Brandes G (2005) Phagocytosis of O4+ axonal fragments in vitro by p75- neonatal rat olfactory ensheathing cells. Glia 49:577-587.

Williams SK, Franklin RJ, Barnett SC (2004) Response of olfactory ensheathing cells to the degeneration and regeneration of the peripheral olfactory system and the involvement of the neuregulins. J Comp Neurol 470:50-62.

Windus LC, Claxton C, Allen CL, Key B, St John JA (2007) Motile membrane protrusions regulate cell-cell adhesion and migration of olfactory ensheathing glia. Glia 55:17081719.

Windus LC, Chehrehasa F, Lineburg KE, Claxton C, Mackay-Sim A, Key B, St John JA (2011) Stimulation of olfactory ensheathing cell motility enhances olfactory axon growth. Cell Mol Life Sci 68:3233-3247. 


\section{Figure Legends}

Figure 1. OMP-ZsGreen x S100ß-DsRed mice provide distinct visualization of immature olfactory sensory neurons and olfactory ensheathing cells (OECs). Panels show laser scanning confocal microscopy (LSCM) images of sagittal sections of the olfactory system of OMP-ZsGreen x S100ß-DsRed mice, dorsal is to the top and rostral to the left. (A) At E11.5, ZsGreen was expressed by primary olfactory neurons within the olfactory epithelium (OE) that lines the caudal recess of the developing nasal cavity (NC). (B-D) In a higher magnification view of the $\mathrm{OE}$ at E11.5, immunostaining with antibodies against Gap43 shows that olfactory sensory neurons expressed Gap43; (C) the majority of the olfactory sensory neurons also expressed ZsGreen in their cell bodies, dendrites and axons (arrow with tail). Some neurons had only low levels (arrow) or no detectable expression (arrowhead) of ZsGreen; merge is shown in D. (E-F) At E13.5, the majority of olfactory sensory neurons expressed ZsGreen and Gap43, although some neurons did not have detectable levels of ZsGreen (arrowhead). The ZsGreen provided distinct visualization of the axons (arrow with tail). (G-I) In E14.5 embryos of OMP-ZsGreen x S100ß-DsRed mice, the DsRed-expressing OECs surrounded the fascicles of ZsGreen olfactory sensory axons; DsRed OECs alone are shown in $\mathrm{H}$; immunostaining with antibodies against p75ntr (I) labelled the OECs. Tel, Telencephalon. Scale bar is $200 \mu \mathrm{m}$ in A, $40 \mu \mathrm{m}$ in B-D, $50 \mu \mathrm{m}$ in E-F, $17 \mu \mathrm{m}$ in G-I. Image

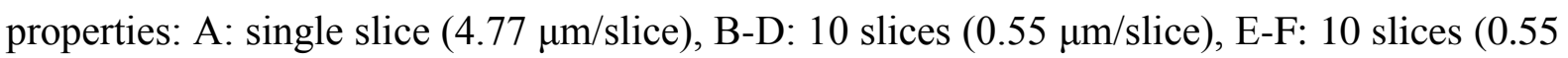
$\mu \mathrm{m} /$ slice $)$, G-I: 16 slices $(0.51 \mu \mathrm{m} /$ slice $)$.

Figure 2. Olfactory ensheathing cells (OECs) phagocytose olfactory axon debris during embryonic development. Panels show LSCM images of sagittal sections of the olfactory system of OMP-ZsGreen x S100ß-DsRed mice, dorsal is to the top and rostral to the left. (A) 
At E13.5, primary olfactory axons projected from the olfactory epithelium (OE) to the developing olfactory bulb (OB). (B) Axon targeting was inaccurate and numerous axons over-projected (arrow) into the deeper layers of the OB. Small isolated fragments of ZsGreen were occasionally present along the nerve fascicles (arrow with tail). Migrating neurons were present (arrowhead) in the migratory mass. (C) At E14.5, the nerve fiber layer (NFL) was established and extended dorsally around the OB. Boxed area is shown in D; asterisk region is shown in E. (D) Higher magnification view of the NFL reveals that ZsGreen olfactory sensory axons (arrow) over-projected into the deeper layers of the developing OB. Small fragments of axons (arrowhead) were also present. (E) In axon fascicles of the lamina propria (asterisk in $\mathrm{C}$ shows location), the DsRed OECs that surrounded the axons contained accumulations of ZsGreen debris (arrows) while some debris was external to OECs (arrowhead); three-dimensional reconstruction is shown in Supplemental movie 1. (F-G) At E15.5, numerous ZsGreen primary olfactory axons over-projected into the deeper layers of the OB (arrow in G); DsRed OECs surrounded the axon fascicles from the OE to the NFL. (H) Within the lamina propria (LP) fragmented axonal debris (dots) was present within the axon fascicles with some debris localized within OECs (arrows); arrowhead points to migrating neuron. (I) Similarly, within the NFL ZsGreen axonal debris (arrow) was internalized within OECs; 3D reconstruction shown in Supplemental movie 2. (J) At E17.5, numerous axons (arrow) over-projected into the deeper layers of the OB. (K-L) Within an axon fascicle of the LP (K) and NFL (L), ZsGreen axonal debris (dots, arrows) was internalized within OECs (arrowhead in L). Nuclei were stained with DAPI in some sections. Scale bar is $500 \mu \mathrm{m}$ in $\mathrm{A}, 100 \mu \mathrm{m}$ in $\mathrm{B}, 290 \mu \mathrm{m}$ in $\mathrm{C}, 25 \mu \mathrm{m}$ in $\mathrm{D}, 10 \mu \mathrm{m}$ in $\mathrm{E}, 275 \mu \mathrm{m}$ in $\mathrm{F}$, $45 \mu \mathrm{m}$ in $\mathrm{G}, 10 \mu \mathrm{m}$ in $\mathrm{H}, 6.5 \mu \mathrm{m}$ in $\mathrm{I}, 80 \mu \mathrm{m}$ in $\mathrm{J}, 14 \mu \mathrm{m}$ in $\mathrm{K}, 6 \mu \mathrm{m}$ in L. Image properties: $\mathrm{A}$ : single slice (4.77 $\mu \mathrm{m} / \mathrm{slice}), \mathrm{B}: 13$ slices $(1.16 \mu \mathrm{m} / \mathrm{slice}), \mathrm{C}$ : single slice (4.77 $\mu \mathrm{m} / \mathrm{slice}), \mathrm{D}: 17$ slices $(0.46 \mu \mathrm{m} / \mathrm{slice}), \mathrm{E}: 15$ slices $(0.52 \mu \mathrm{m} / \mathrm{slice}), \mathrm{F}$ : single slice $(4.77 \mu \mathrm{m} / \mathrm{slice}), \mathrm{G}: 12$ 
slices $(1.16 \mu \mathrm{m} / \mathrm{slice}), \mathrm{H}: 17$ slices $(0.45 \mu \mathrm{m} / \mathrm{slice}), \mathrm{I}: 11$ slices $(0.45 \mu \mathrm{m} / \mathrm{slice}), \mathrm{J}: 30$ slices (1.16 $\mu \mathrm{m} / \mathrm{slice}), \mathrm{K}: 16$ slices $(0.45 \mu \mathrm{m} / \mathrm{slice}), \mathrm{L}: 32$ slices $(0.38 \mu \mathrm{m} / \mathrm{slice})$.

Figure 3. OECs phagocytose olfactory axon debris during early postnatal development. Panels show LSCM images of coronal sections of postnatal day 3 (P3) OMP-ZsGreen $x$ S100ß-DsRed mice with dorsal to the top. (A) Olfactory region of a P3 mouse; boxed area is shown in B. (B) Primary olfactory axons (arrow) over-projected into the external plexiform layer (EPL). Discontinuous fragmented axons (arrowhead) were also present in the EPL. (C) In axon fascicles projecting from the OE through the LP, accumulations of ZsGreen axonal debris (arrows) were present and coincided with OECs. Cartilage (C) also expressed DsRed protein. (D-E) Higher magnification view of an axon fascicle within the LP showing axonal debris (dots, arrows) accumulated within OECs. (F) Similarly within the NFL, axonal debris (dots) was internalized by OECs. For these images, the intact axons are barely visible due to the high intensity fluorescence of the axon debris which required lower laser intensity during image acquisition. 3D reconstruction of panel 3F is shown in Supplemental movie 3. Nuclei were stained with DAPI in A and C. Scale bar $=500 \mu \mathrm{m}$ in A, $70 \mu \mathrm{m}$ in B; $25 \mu \mathrm{m}$ in $\mathrm{C}, 10$ $\mu \mathrm{m}$ in D-E, $5 \mu \mathrm{m}$ in F. Image properties: A: single slice $(4.77 \mu \mathrm{m} / \mathrm{slice})$, B: 13 slices $(1.16$ $\mu \mathrm{m} / \mathrm{slice}), \mathrm{C}: 10$ slices $(1.16 \mu \mathrm{m} / \mathrm{slice}), \mathrm{D}-\mathrm{E}: 35$ slices $(0.46 \mu \mathrm{m} / \mathrm{slice}), \mathrm{F}: 17$ slices $(0.46$ $\mu \mathrm{m} /$ slice).

Figure 4. Macrophages are not the primary phagocytic cell in the olfactory nerve during development. Panels A-C show LSCM images of coronal sections of OMP-ZsGreen $\mathrm{x}$ S100ß-DsRed postnatal day 7 pups immunostained with anti-Iba1 antibodies, dorsal is to the top. (A) Macrophages labelled by anti-Iba1 (arrows) were present around axons fascicles in the LP and the exterior of the NFL. Boxed area is shown in C. (B) In axon fascicles within 
the LP macrophages occasionally contained small amounts of axon debris (dot, arrowhead) while OECs frequently contained large amounts of debris (arrow). (C) Similarly, in the NFL macrophages occasionally contained small amounts of axon debris (arrowhead) while adjacent OECs had large accumulations of debris (arrows). Panels D-I show LSCM images of coronal sections of P7 S100ß-DsRed x MacBlue transgenic mice. (D) In axon fascicles of the LP, macrophages (arrowhead) were largely restricted to the exterior of the fascicles. (E) Macrophages (arrowhead) were infrequently present within the NFL. (F-G) Low power image of the OB shows OECs populated the NFL while macrophages were present in the deeper layers of the OB. (H-I) Higher power view of the NFL and EPL. Macrophages (arrowheads) were present in low numbers in the NFL but were numerous within the EPL (arrow). (J) Quantification of the number of macrophages in the NFL and central region of the OB per $200 \times 200 \mu \mathrm{m}$ square region; $\mathrm{n}=3$ animals; ***p<0.001, t-test. Scale bar $=100$ $\mu \mathrm{m}$ in $\mathrm{A}, 11 \mu \mathrm{m}$ in B-C, $25 \mu \mathrm{m}$ in D-E, $200 \mu \mathrm{m}$ in F-G, $60 \mu \mathrm{m}$ in H-I. Image properties: A: 22 slices $(1.26 \mu \mathrm{m} / \mathrm{slice}), \mathrm{B}: 15$ slices $(0.52 \mu \mathrm{m} /$ slice $)$, C: 20 slices $(0.52 \mu \mathrm{m} /$ slice $)$, D: 15 slices $(0.52 \mu \mathrm{m} / \mathrm{slice}),) \mathrm{E}: 14$ slices $(0.52 \mu \mathrm{m} / \mathrm{slice}), \mathrm{F}-\mathrm{G}$ : single slice $(4.77 \mu \mathrm{m} / \mathrm{slice}), \mathrm{H}-\mathrm{I}: 30$ slices $(0.52 \mu \mathrm{m} / \mathrm{slice})$.

Figure 5. Axon debris is internalized within embryonic OECs. Panels show LSCM images of OECs that were dissected out of OMP-ZsGreen x S100ß-DsRed embryos. (A) Dissociated E14.5 DsRed OECs from the LP that were fixed immediately upon attachment to the culture plate contained ZsGreen axon debris (dots). (B-C) Immunostaining of dissociated E14.5 OECs shows that DsRed-expressing OECs that contained ZsGreen axon debris (arrows in B) also expressed p75ntr (C). (D-F) An OEC from an E14.5 embryo $24 \mathrm{~h}$ after dissection and immunostained with anti-lamp1 antibodies showed that ZsGreen debris (arrows in E) colocalized with anti-lamp1 (arrows in D); merge with DsRed is shown in F. (G-H) A 
dissociated DsRed OEC from an E15.5 embryo had numerous accumulations of ZsGreen debris within the cell body (arrows in G) and along the shaft of the process (arrowheads in H). Scale bar $=20 \mu \mathrm{m}$ in A, $15 \mu \mathrm{m}$ in B-C, $6 \mu \mathrm{m}$ in D-F, $18 \mu \mathrm{m}$ in G-H. Image properties: A: 4 slices $(0.5 \mu \mathrm{m} / \mathrm{slice}), \mathrm{B}-\mathrm{C}: 8$ slices $(0.46 \mu \mathrm{m} / \mathrm{slice}), \mathrm{D}-\mathrm{F}: 4$ slices $(0.52 \mu \mathrm{m} / \mathrm{slice}), \mathrm{G}-\mathrm{H}: 6$ slices $(0.52 \mu \mathrm{m} / \mathrm{slice})$.

Figure 6. OECs phagocytose axon debris in vitro. (A-B) Dissociated cultures of embryonic OECs dissected from the septum of S100ß-DsRed E14 embryos; (A) epifluorescent image showing DsRed OECs $18 \mathrm{~h}$ after plating had a largely bipolar morphology and numerous lamellipodia (arrowheads); (B) LSCM image of live OECs to which exogenous ZsGreen fluorescent debris had been added to the medium and then internalized (arrowheads) by the OECs. Time after addition of debris is in h:min. (C-D) Scanning electron micrograph of (C) untreated OECs, (D) OECs 15 min after addition of axon debris. Numerous pseudopodial protrusions (arrow) extended from the OECs in response to the debris. (E) Quantification of the number of protrusions per cell body, $\mathrm{p}<0.001(* * *)$, two-way analysis of variance with Tukey posthoc test, $\mathrm{n}=8-16$ per treatment. Scale bar $=25 \mu \mathrm{m}$ in $\mathrm{A}, 16 \mu \mathrm{m}$ in $\mathrm{B}, 2 \mu \mathrm{m}$ in $\mathrm{C}$ D. Fluorescent image properties: A: epifluorescent image; B: 9 slice Z-stack $(0.52 \mu \mathrm{m} / \mathrm{slice})$.

Figure 7. OECs phagocytose olfactory axon debris after widespread degeneration. Panels show LSCM images of axon fascicles in an animal that had undergone unilateral bulbectomy at postnatal day 4 and examined at postnatal day 7. (A) Panel shows coronal section through the rostral region of the OBs. One OB was ablated (asterisk shows location) and neurons in the OE degenerated; the other OB was intact. (B) Higher magnification view of the OECs within the axon fascicles of the LP on the unablated side; the fascicle indicated by the asterisk is shown in C-D. (C-D) Cross sectional view of an axon fascicle shows the morphology of 
the OECs and that some ZsGreen axon debris (arrow) was contained within OECs. Numerous extensive lamellipodia of the OECs penetrated all regions of the fascicle. 3D reconstruction of $\mathrm{C}$ is shown in Supplemental movie 4. (E) High magnification of a small region of an axon fascicle showed the extensive lamellipodial protrusions throughout the fascicle as well as the accumulation of some axon debris (green dots) within OECs. 3D reconstruction of $\mathrm{E}$ is shown in Supplemental movie 5. (F) On the bulbectomized side of the same animal as shown in B-E, the neurons within the OE were degraded and there were large accumulations of ZsGreen axon debris (arrow) within the OECs of the axon fascicles. (G) Morphology of the OECs shows that while the OECs (arrowhead) maintained their ensheathment of the fascicles, the lamellipodia within the fascicles had retracted leaving open voids (arrows). Fascicle indicated by the asterisk is shown in H-I. (H-I) Higher magnification of a fascicle shows the large accumulation of axon debris within OECs and the loss of OEC lamellipodia from many areas of the internal regions of the fascicle (arrow in $\mathrm{H}$ ). 3D reconstruction of $\mathrm{H}$ is shown in Supplemental movie 6. (J) Higher magnification of a region of a fascicle shows accumulation of axon debris (green dots) within OECs and the more open structure of the OEC lamellipodia morphology. 3D reconstruction of $\mathbf{J}$ is shown in Supplemental movie 7. Scale bar $=500 \mu \mathrm{m}$ in $\mathrm{A}, 40 \mu \mathrm{m}$ in $\mathrm{B}, \mathrm{G}, 19 \mu \mathrm{m}$ in $\mathrm{C}-\mathrm{D}, 6.5 \mu \mathrm{m}$ in $\mathrm{E}, \mathrm{J}, 85 \mu \mathrm{m}$ in $\mathrm{F}, 16 \mu \mathrm{m}$ in H-I. Image properties: A: single slice $(4.77 \mu \mathrm{m} / \mathrm{slice}), \mathrm{B}: 7$ slices, $(1.16 \mu \mathrm{m} / \mathrm{slice}), \mathrm{C}-\mathrm{D}: 7$ slices $(1.16 \mu \mathrm{m} /$ slice $)$, E: 20 slices $(0.52 \mu \mathrm{m} / \mathrm{slice}), \mathrm{F}: 4$ slices $(1.26 \mu \mathrm{m} / \mathrm{slice}), \mathrm{G}: 7$ slices, (1.16 $\mu \mathrm{m} /$ slice), H-I: 18 slices $(0.52 \mu \mathrm{m} /$ slice $), \mathrm{J}: 20$ slices, $(0.52 \mu \mathrm{m} / \mathrm{slice})$.

Figure 8. Macrophages are not the principal phagocytic cells after widespread degeneration. Panels show LSCM images of coronal sections of animals that underwent bulbectomy (A-B) and methimazole treatment (C-D). (A) Three days after unilateral bulbectomy (OBX), neurons and their axons degenerated in the OE; the contralateral $\mathrm{OB}$ and $\mathrm{OE}$ remained intact. 
Arrow points to region shown in panel B. (B) Higher magnification view of an olfactory nerve fascicle within the LP on the OBX side immunostained with antibodies against Ibal to visualise macrophages. The majority of ZsGreen axonal debris (dots) was contained within OECs but some macrophages did contain some axonal debris (arrowheads). (C-D) Postnatal day 3 pups treated with methimazole and then examined at P7. The majority of neurons had degenerated and numerous axon debris (dots) was present in the OE and LP. (D) Higher magnification view of an axon fascicle showed that most debris was contained within OECs (arrows), while macrophages (arrowhead) contained little or no debris. Scale bar $=500 \mu \mathrm{m}$ in $\mathrm{A}, 18 \mu \mathrm{m}$ in $\mathrm{B}, 50 \mu \mathrm{m}$ in $\mathrm{C}, 13 \mu \mathrm{m}$ in D. Image properties: A: single slice (4.77 $\mu \mathrm{m} / \mathrm{slice})$, B: 17 slices $(0.52 \mu \mathrm{m} / \mathrm{slice}), \mathrm{C}: 16$ slices, $(1.26 \mu \mathrm{m} / \mathrm{slice}), \mathrm{D}: 18$ slices $(0.52 \mu \mathrm{m} / \mathrm{slice})$. 